SPhT-T03/121

hep-th/0308205

CPHT-RR-051-0803

\title{
On Minisuperspace Models of S-branes
}

\author{
Stefan Fredenhagen ${ }^{1}$ and Volker Schomerus ${ }^{2}$ \\ ${ }^{1}$ CPHT - Ecole Polytechnique, \\ F-91128 Palaiseau CEDEX, France \\ 2 Service de Physique Théorique, CEA Saclay, \\ F-91191 Gif-sur-Yvette CEDEX, France
}

August 29, 2003

\begin{abstract}
In this note we reconsider the minisuperspace toy models for rolling and bouncing tachyons. We show that the theories require to choose boundary conditions at infinity since particles in an exponentially unbounded potential fall to infinity in finite world-sheet time. Using standard techniques from operator theory, we determine the possible boundary conditions and we compute the corresponding energy spectra and minisuperspace 3-point functions. Based on this analysis we argue in particular that world-sheet models of S-branes possess a discrete spectrum of conformal weights containing both positive and negative values. Finally, some suggestions are made for possible relations with previous studies of the minisuperspace theory.
\end{abstract}

e-mail: stefan@cpht.polytechnique.fr vschomer@spht.saclay.cea.fr 


\section{Introduction}

Time dependent open and closed string backgrounds have received a lot of attention during the last year. Some of the initial motivation was rooted in the search for a dS/CFT correspondence from which the notion of S-branes emerged [1]. Their world-sheet description remained obscure until A. Sen proposed [2, 3] to construct them by adding a $\cosh X_{0}$-shaped boundary interaction to the free time-like bosonic field theory. A very closely related theory with a Liouville-like $\exp X_{0}$ boundary potential was first studied by Strominger [4] in a minisuperspace approximation. It describes a half-brane, i.e. a brane that decays as time evolves.

Our knowledge about these two world-sheet theories is still quite limited. Most of the recent work centered around their boundary states (see e.g. [2, 3, 5, 6, 17, 8, 9, 10, ). The latter were obtained through analytic continuation ('Wick-rotation') from Euclidean theories with a $\cos X$ or $\exp i X$ boundary potential, respectively. Other quantities, such as the boundary 2- and 3-point functions or bulk-boundary 2-point functions, have not been computed (some proposal for the boundary 2-point function can be found in [11]). But in the light of the analysis in [12, one outcome of a full construction appears to be certain: most quantities in these two backgrounds cannot be obtained through Wickrotation simply because higher correlators in the Euclidean models are no longer analytic. ${ }^{1}$

Unfortunately, general tools for the direct construction of 2-dimensional world-sheet models on non-trivial time-like or Lorentzian space-times have not been developed. On the other hand, technology for the analysis of the corresponding minisuperspace models is certainly available and in fact fairly standard. Hence, investigations of such toy particle models do not require to pass through the corresponding Euclidean background. Nevertheless they can still cast some light on the structure of the more complicated field theory models. We take this as a motivation to re-investigate the time-like minisuperspace toy models for rolling and bouncing tachyons. Our analysis uncovers that the Lorentzian models have features which are very distinct from their Euclidean counterparts. In particular, parameters emerge that do not appear in the classical action and the spectra of world-sheet Hamiltonians possess new branches of discrete eigenvalues.

\footnotetext{
${ }^{1}$ Though it might be possible exploit the same trick as in 12 where a non-analytic $c=1$ model related to closed string tachyon decay was embedded into a family of analytic theories with $c \geq 1$.
} 
In the case of the rolling tachyon theory much of the relevant mathematical results were previously derived in [13, 14], obviously with different applications in mind. Below we shall review and extend these findings. Several arguments will be presented showing that a consistent quantum theory requires to choose boundary conditions in the far future. More precisely, wave functions $\psi$ of the rolling tachyon model

$$
H_{\mathrm{rt}}=\partial_{x_{0}}^{2}+\lambda e^{2 x_{0}} \quad \text { with } \quad \lambda>0
$$

must behave asymptotically as

$$
\psi\left(x_{0}\right) \stackrel{x_{0} \rightarrow \infty}{\sim} \text { const. } \cdot e^{-x_{0} / 2} \cos \left(\sqrt{\lambda} e^{x_{0}}-\frac{\pi}{4}-\pi \nu_{0}\right)
$$

where $\nu_{0} \in(0,1]$ is some real parameter. The extension of $H_{\mathrm{rt}}$ to wave functions with such asymptotics is necessary to turn $H_{\mathrm{rt}}$ into a consistent, self-adjoint Hamilton operator in the 'world-sheet' theory. After we have chosen such an extension, we can look for eigenfunctions and determine the spectrum of eigenvalues $\Delta$ of the rolling tachyon model. Explicit formulas for the eigenfunctions are provided in section 2 (see eqs. (2.52.6)). The associated spectra depend on the boundary conditions $\nu_{0}$ and consist of a continuous and a discrete branch. More precisely, we shall find

$$
\operatorname{Spec}_{\nu_{0}}\left(H_{\mathrm{rt}}\right)=(-\infty, 0] \cup \bigcup_{n=0}^{\infty}\left\{4\left(\nu_{0}+n\right)^{2}\right\} .
$$

The answer resembles the spectrum of a Schrödinger problem on a half-line. Hence, one may conclude that the coordinate $x_{0}$ is somehow cut off in the future. This effect can actually be traced back to the structure of solutions in the associated classical model. In fact, it is not hard to see that a particle in an exponentially decreasing potential $V\left(x_{0}\right)=-\lambda e^{2 x_{0}}$ falls to $x_{0}=\infty$ in finite 'world-sheet' time.

These findings differ significantly from the minisuperspace analysis of Strominger [4] (see also [15, 16]). Strominger's toy model arises from a continuation of the Euclidean theory and consequently the parameter $\nu_{0}$ does not appear. To investigate the relation between the two approaches, we shall compute the minisuperspace analogues of the 2- and 3 -point functions in our framework. Integration over the parameter $\nu_{0}$ is then shown to lead to the corresponding quantities in Strominger's minisuperspace model (see [4, 12]).

Similar results are also derived for the bouncing tachyon Hamiltonian (see e.g. 17, 18] for previous studies of the minisuperspace model in the context of tachyon dynamics)

$$
H_{\mathrm{bt}}=\partial_{x_{0}}^{2}+2 \lambda \cosh 2 x_{0} \text {. }
$$


In this case, there exists a 4-parameter family of extensions, but only two parameters remain if we require that the far future is not coupled to the far past. The corresponding wave functions possess the same asymptotics as for the rolling tachyon, i.e.

$$
\psi\left(x_{0}\right) \stackrel{x_{0} \rightarrow \pm \infty}{\sim} \text { const. } \cdot e^{-\left|x_{0}\right| / 2} \cos \left(\sqrt{\lambda} e^{\left|x_{0}\right|}-\frac{\pi}{4}-\pi \nu_{ \pm}\right)
$$

with two real parameters $\nu_{ \pm} \in(0,1]$. For all choices of the boundary parameters, the spectrum of eigenvalues $\Delta$ is purely discrete. We shall not construct it explicitly, but we shall be able to make two statements about its behaviour for $|\Delta| \rightarrow \infty$. The first one concerns the allowed eigenvalues for $\Delta \gg 2 \lambda$,

$$
\operatorname{Spec}_{\nu_{ \pm}}\left(H_{\mathrm{bt}}\right) \cap\left\{\Delta>4 N^{2}\right\} \sim \bigcup_{n=N}^{\infty}\left\{4\left(\nu_{+}+n\right)^{2}\right\} \cup \bigcup_{n=N}^{\infty}\left\{4\left(\nu_{-}+n\right)^{2}\right\} \quad \text { for } \quad N \rightarrow \infty .
$$

On the other side, when $-\Delta$ becomes very large we can show that the distance $\delta \Delta$ between two consecutive spectral lines behaves as

$$
\delta \Delta \sim \frac{2 \pi \sqrt{-\Delta}}{\log (-4 \Delta / \lambda)} \text { for } \quad \Delta \rightarrow-\infty
$$

The explanation of these results and their consequences are similar to the discussion for the rolling tachyon background.

Our presentation begins with the rolling tachyon model. After a brief study of its classical solutions we recall some simple facts from operator theory that are needed for the analysis of the Hamiltonian in the quantum theory. We then spell out and prove very explicit formulas for the wave functions, the spectrum and the minisuperspace analogue of the 3-point functions. The bouncing tachyon model is addressed in section 3 . In this case, the expressions we arrive at are a little less explicit, but they are sufficient to establish the picture we have sketched above.

\section{The rolling tachyon model}

In this section we investigate the minisuperspace toy model of a rolling tachyon background. In order to understand the necessity of imposing boundary conditions at infinity, we begin with some remarks on classical solutions. Then we analyse the admissible boundary conditions in the quantum theory and we determine the spectra of the associated models. Finally, we compute the minisuperspace analogues of the 3-point function and conclude with some remarks about their relation with quantities calculated in 4, 12. 


\subsection{Classical solutions of the rolling tachyon model}

The classical action of the open string rolling tachyon background on a Lorentzian worldsheet $\Sigma=\mathbb{R} \times[0, \pi]$ takes the form

$$
S_{\mathrm{RT}}^{o}=-\frac{1}{4 \pi} \int_{\Sigma} d \sigma d t\left(\partial_{t} X_{0} \partial_{t} X_{0}-\partial_{\sigma} X_{0} \partial_{\sigma} X_{0}\right)-\lambda^{o} \int d t e^{X_{0}} .
$$

Throughout this note we set $\alpha^{\prime}=1$ and the constant $\lambda^{o}$ is assumed to be positive. The associated minisuperspace toy model is obtained by replacing the world-sheet field $X_{0}$ with a map $\tilde{x}_{0}=\tilde{x}_{0}(t)$ which is independent of the world-sheet coordinate $\sigma$,

$$
S_{\mathrm{rt}}^{o}=-\int_{-\infty}^{\infty} d t\left(\frac{1}{4} \partial_{t} \tilde{x}_{0} \partial_{t} \tilde{x}_{0}+\lambda^{o} e^{\tilde{x}_{0}}\right) .
$$

It is not difficult to find classical solutions of this model. They are parametrised by two real parameters $\Delta^{o}$ and $t_{f}$. The explicit form of the solutions depends on whether $\Delta^{o}$ is positive or negative. For $\Delta^{o} \leq 0$ we find

$$
e^{\tilde{x}_{0}(t)}=-\frac{\Delta^{o}}{\lambda^{o}} \frac{4 \exp 2 \sqrt{-\Delta^{o}}\left(t-t_{f}\right)}{\left(1-\exp 2 \sqrt{-\Delta^{o}}\left(t-t_{f}\right)\right)^{2}},
$$

while solutions with positive $\Delta^{o}>0$ can be written in the form

$$
e^{\tilde{x}_{0}(t)}=\frac{\Delta^{o}}{\lambda^{o}} \sin ^{-2} \sqrt{\Delta^{o}}\left(t-t_{f}\right)
$$

A short computation shows that both expressions correspond to stationary points of $S_{\text {rt }}^{o}$. The parameters $\Delta_{o}$ and $t_{f}$ are fixed by the initial conditions at $t=0 . \Delta^{o}$ may be interpreted as the 'world-sheet' energy. The parameter $t_{f}$, on the other hand, is the 'world-sheet' time at which we reach $\tilde{x}_{0}=\infty$. Note that for all finite choices of initial conditions, the time $t_{f}$ is finite. In other words, the dynamics of the rolling tachyon model implies that the far future $\tilde{x}_{0}=\infty$ is reached in finite 'world-sheet' time. Experience with similar models suggests that we shall have to impose some boundary condition at $\tilde{x}_{0}=\infty$ in order to make the associated quantum theory well defined. We shall show shortly that this is indeed the case.

Let us also briefly comment on the minisuperspace model for the closed string rolling tachyon background. The latter is derived from the corresponding action

$$
S_{\mathrm{RT}}^{c}=-\frac{1}{4 \pi} \int_{\Sigma} d \sigma d t\left(\partial_{t} X_{0} \partial_{t} X_{0}-\partial_{\sigma} X_{0} \partial_{\sigma} X_{0}+4 \lambda e^{2 X^{0}}\right) .
$$


Reduction to maps $x_{o}(t)$ which are independent of $\sigma$ gives the following minisuperspace toy model for the closed string theory

$$
S_{\mathrm{rt}}^{c}=-\int_{-\infty}^{\infty} d t\left(\frac{1}{4} \partial_{t} x_{0} \partial_{t} x_{0}+\lambda e^{2 x_{0}}\right)
$$

Note that this is essentially the same model as in the case of open strings. In fact, the two theories can be rewritten into each other by means of the replacement rules $\tilde{x}_{0} \leftrightarrow 2 x_{0}$, $\lambda^{o} \leftrightarrow 4 \lambda$ and $\Delta^{o} \leftrightarrow 4 \Delta$ where $\Delta$ is the world-sheet energy in the closed string model. Since some of the formulas below are a bit simpler when written in terms of the closed string parameters, we shall use them from now on. Rewriting our results for the open string model is trivial.

\subsection{Spectral analysis for the rolling tachyon}

Our aim now is to study the following toy model Hamiltonian for the rolling tachyon background,

$$
H_{\mathrm{rt}}=\partial_{x_{0}}^{2}+\lambda e^{2 x_{0}} \quad \text { with } \quad \lambda>0
$$

This Hamiltonian should be regarded as the minisuperspace analogue of the operator $L_{0}+\bar{L}_{0}$ (or $L_{0}$ when we deal with open strings) in the conformal field theory of rolling tachyons. In both the conformal field theory and its toy model, the Hamiltonian is the generator of 'world-sheet' time translations and therefore it has to be be self-adjoint.

Our Hamiltonian $H_{\mathrm{rt}}$ is originally defined on the space of smooth functions with compact support and while it is not self-adjoint on this domain, it is easily seen to admit a 1-parameter family of self-adjoint extensions. For each of these extensions, the spectrum of eigenvalues and the associated eigenfunctions have been determined in [13, 14. The possible eigenvalues are toy model analogues of the scaling dimensions in the conformal field theory. Their spectrum turns out to be continuous for negative eigenvalues. ${ }^{2}$ On the other hand, discrete positive eigenvalues can appear. Furthermore, there is only a single eigenfunction for each allowed eigenvalue, just as in spectral problems on the half-line. In this sense, the theory appears as if it was cut off in the far future, thereby reflecting the properties of the classical system that we discussed in the previous subsection.

Even though all these results can be found in the cited literature, we shall briefly go through their derivation, mainly to prepare for the analysis of the bouncing tachyon

\footnotetext{
${ }^{2}$ Note that we have changed the sign of the Hamiltonian in comparison to [13, 14].
} 
model. Let us therefore begin by showing that there is indeed a 1-parameter family of self-adjoint extensions. According to standard operator theory (see e.g. [19]), we are supposed to determine the so-called deficiency indices of $H_{\mathrm{rt}}$, i.e. we have to determine the number of independent square-integrable solutions for each of the two differential equations $H_{\mathrm{rt}} \psi^{ \pm}= \pm i \psi^{ \pm}$. The problem is fairly easy to analyse. In fact, for each sign, the equation certainly has two linearly independent solutions which we can choose as

$$
\psi_{1}^{ \pm}\left(x_{0}\right)=J_{\eta^{ \pm}}\left(\sqrt{\lambda} e^{x_{0}}\right) \text { and } \psi_{2}^{ \pm}\left(x_{0}\right)=J_{-\eta^{ \pm}}\left(\sqrt{\lambda} e^{x_{0}}\right)
$$

with $\eta^{+}=\exp (3 \pi i / 4)$ and $\eta^{-}=\exp (\pi i / 4)$. Here, $J_{\nu}$ is a Bessel function of the first kind. Among these four functions, two are not square-integrable because they diverge exponentially in the far past $x_{0} \rightarrow-\infty$. Hence, only the functions $\psi_{1}^{-}$and $\psi_{2}^{+}$satisfy our search criteria. Since there is one such function for each sign in the differential equation, the deficiency indices are $(1,1)$. This tells us that there exists indeed a 1-parameter family of self-adjoint extensions as we have claimed above.

We shall denote the corresponding (real) parameter by $\nu_{0}$ and the domains of the associated self-adjoint extensions by $D_{\nu_{0}}\left(H_{\mathrm{rt}}\right)$. The direct approach to finding the spectrum of these extensions is to construct the domains $D_{\nu_{0}}\left(H_{\mathrm{rt}}\right)$ and to diagonalize $H_{\mathrm{rt}}$ thereon. Since the construction of the domains is a bit formal, we shall postpone this issue and start with a somewhat simpler and more intuitive analysis of the spectrum by looking for a 'maximal set of compatible eigenfunctions'.

To this end, let us assume that $H_{\mathrm{rt}}$ has been extended to one of the domains $D_{\nu_{0}}\left(H_{\mathrm{rt}}\right)$. On each domain, the operator $H_{\mathrm{rt}}$ is symmetric, i.e.

$$
\left\langle\psi \mid H_{\mathrm{rt}} \psi^{\prime}\right\rangle=\left\langle H_{\mathrm{rt}} \psi \mid \psi^{\prime}\right\rangle \quad \text { for all } \quad \psi, \psi^{\prime} \in D_{\nu_{0}}\left(H_{\mathrm{rt}}\right)
$$

If we insert the explicit form of $H_{\mathrm{rt}}$, this property can be re-expressed as a boundary condition for functions in the domain,

$$
\left.\left(\bar{\psi}\left(x_{0}\right) \partial_{x_{0}} \psi^{\prime}\left(x_{0}\right)-\psi^{\prime}\left(x_{0}\right) \partial_{x_{0}} \bar{\psi}\left(x_{0}\right)\right)\right|_{-\infty} ^{\infty}=0 \quad \text { for all } \quad \psi, \psi^{\prime} \in D_{\nu_{0}}\left(H_{\mathrm{rt}}\right)
$$

The different domains we can select for self-adjoint extensions of $H_{\mathrm{rt}}$ correspond to different choices of boundary conditions at infinity. All of these boundary conditions have to be consistent with the previous equation. This provides us with some sort of compatibility condition which two functions from the same domain $D_{\nu_{0}}\left(H_{\mathrm{rt}}\right)$ have to satisfy. 
We can use this condition to find sets of compatible eigenfunctions $\psi_{\Delta}^{\nu_{0}}$ of $H_{\mathrm{rt}}$, i.e. compatible solutions of the differential equation

$$
H_{\mathrm{rt}} \psi_{\Delta}^{\nu_{0}}\left(x_{0}\right)=\Delta \psi_{\Delta}^{\nu_{0}}\left(x_{0}\right) \quad \text { for } \quad \psi_{\Delta}^{\nu_{0}} \in D_{\nu_{0}}\left(H_{\mathrm{rt}}\right)
$$

As we shall see momentarily, the boundary condition (2.3) applied to an arbitrary pair of eigenfunctions turns into a powerful constraint, both on the allowed eigenvalues and on the form of the eigenfunctions.

To begin with, let us study the situation where $\Delta=4 \nu^{2}$ is positive. In this case, one of the Bessel functions that solves the differential equation (2.4) diverges exponentially in the far past so that eigenfunctions of $H_{\mathrm{rt}}$ are necessarily of the form

$$
\psi_{\nu}^{\nu_{0}}\left(x_{0}\right)=2 \sqrt{\nu} J_{2 \nu}\left(\sqrt{\lambda} e^{x_{0}}\right) \text { for } \nu>0
$$

where the prefactor is chosen s.t. $\left\|\psi_{\nu}^{\nu_{0}}\right\|_{2}=1$. Using the asymptotic behaviour of Bessel functions for large arguments, it is then easy to see that the condition (2.3) for two such functions $\psi_{\nu}^{\nu_{0}}, \psi_{\nu^{\prime}}^{\nu_{0}}$ to be in the domain of the same self-adjoint extension becomes

$$
\sin \pi\left(\nu-\nu^{\prime}\right)=0
$$

This means that $\nu-\nu^{\prime} \in \mathbb{Z}$ or, equivalently, that the only allowed values for $\nu$ are given by $\nu_{n}=n+\nu_{0}, n=0,1,2, \ldots$. Here we have associated our parameter $\nu_{0} \in(0,1]$ with the smallest positive eigenvalue that can appear. Our argument shows that for positive $\Delta$, the spectrum of $H_{\mathrm{rt}}$ on the domain $D_{\nu_{0}}\left(H_{\mathrm{rt}}\right)$ is discrete with eigenvalues of the form $\Delta_{n}=4\left(n+\nu_{0}\right)^{2}$.

Now we want to extend the analysis to include negative eigenvalues. In evaluating our condition (2.3) we encounter a potential problem because $\psi_{\Delta}\left(x_{0}\right)$ with $\Delta \leq 0$ approaches a free wave for $x_{0} \rightarrow-\infty$ : it is not square-integrable and thus not in $D_{\nu_{0}}\left(H_{\mathrm{rt}}\right)$. The issue is resolved by smearing eigenfunctions with a smooth energy distribution $\rho(\Delta)$. No matter how sharply peaked this distribution is, the resulting smeared wave function and its derivative will vanish for $x_{0} \rightarrow-\infty$ due to the fact that the eigenfunctions oscillate faster and faster in the parameter $\Delta$ as we approach the far past $x_{0} \rightarrow-\infty$. On the other side, for $x_{0} \rightarrow \infty$ the eigenfunctions do not show this oscillating behaviour in $\Delta$ so that their asymptotics can be approximated arbitrarily closely by smeared wave functions. This means that we are allowed to evaluate the condition (2.3) at $x_{0}=\infty$ also for eigenfunctions associated to the negative spectrum. 
Let us now choose $\psi=\psi_{\nu_{n}}^{\nu_{0}}$ and let $\psi_{\Delta}^{\prime}$ be an eigenfunction for non-positive eigenvalue $\Delta$. For the latter, we make the general Ansatz

$$
\psi_{\Delta}^{\prime}\left(x_{0}\right)=\alpha_{\Delta} J_{-i \sqrt{-\Delta}}\left(\sqrt{\lambda} e^{x_{0}}\right)+\beta_{\Delta} J_{i \sqrt{-\Delta}}\left(\sqrt{\lambda} e^{x_{0}}\right)
$$

Applying the condition (2.3) to $\psi$ and $\psi^{\prime}$ then gives

$$
\alpha_{\Delta} \sinh \pi\left(\omega-i \nu_{0}\right)=\beta_{\Delta} \sinh \pi\left(\omega+i \nu_{0}\right)
$$

where $\Delta=-4 \omega^{2}$. This implies that there exists only one eigenfunction for each value of $\Delta \leq 0$. We can take it to be of the form

$$
\psi_{\omega}^{\nu_{0}}\left(x_{0}\right)=(2 \omega / \sinh 2 \pi \omega)^{\frac{1}{2}}\left(J_{-2 i \omega}\left(\sqrt{\lambda} e^{x_{0}}\right)+\frac{\sinh \pi\left(\omega-i \nu_{0}\right)}{\sinh \pi\left(\omega+i \nu_{0}\right)} J_{2 i \omega}\left(\sqrt{\lambda} e^{x_{0}}\right)\right) .
$$

It is finally simple to check the condition (2.3) at $x_{0}=\infty$ for any pair of functions (2.6). This is in fact equivalent to the orthogonality of the system of eigenfunctions $\psi_{\omega}^{\nu_{0}}$,

$$
\int_{-\infty}^{\infty} d x_{0} \overline{\psi_{\omega}^{\nu_{0}}}\left(x_{0}\right) \psi_{\omega^{\prime}}^{\nu_{0}}\left(x_{0}\right)=\delta\left(\omega-\omega^{\prime}\right)
$$

We thus arrive at a family of 'maximal sets of compatible eigenfunctions'. The spectrum and the corresponding wave functions are sketched in figure 1] To be sure that we did not overlook any part of the spectrum, we can verify completeness by showing that

$$
\int_{0}^{\infty} d \omega \overline{\psi_{\omega}^{\nu_{0}}}\left(x_{0}\right) \psi_{\omega}^{\nu_{0}}\left(x_{0}^{\prime}\right)+\sum_{n=0}^{\infty} \overline{\psi_{\nu_{n}}^{\nu_{0}}}\left(x_{0}\right) \psi_{\nu_{n}}^{\nu_{0}}\left(x_{0}^{\prime}\right)=\delta\left(x_{0}-x_{0}^{\prime}\right)
$$

While the proof of the orthogonality is left as an exercise, details on the derivation of eq. (2.7) can be found in appendix A. We would also like to stress that all the eigenfunctions we found for a given parameter $\nu_{0}$ possess the same asymptotics (1.2) in the far future.

This finally brings us back to the direct construction of the domains $D_{\nu_{0}}\left(H_{\mathrm{rt}}\right)$ involving the functions $\psi_{1}^{-}$and $\psi_{2}^{+}$. The general prescription due to J. von Neumann [20] is to first take the domain $D\left(\bar{H}_{\mathrm{rt}}\right)$ of the closure $\bar{H}_{\mathrm{rt}}$ of the original operator $H_{\mathrm{rt}}$ defined on the smooth functions with compact support. Then the domain $D_{\nu^{\prime}}\left(H_{\mathrm{rt}}\right)$ of an extension labeled by some parameter $\nu^{\prime} \in(0,1]$ is given by

$$
D_{\nu^{\prime}}\left(H_{\mathrm{rt}}\right)=\left\{\psi+\alpha\left(\psi_{1}^{-}+e^{2 \pi i \nu^{\prime}} \psi_{2}^{+}\right) \mid \psi \in D\left(\bar{H}_{\mathrm{rt}}\right), \alpha \in \mathbb{C}\right\}
$$

It can be seen that $D\left(\bar{H}_{\mathrm{rt}}\right)$ does not contain any of the eigenfunctions of $H_{\mathrm{rt}}$. In fact, the latter fall off like $e^{-x_{0} / 2}$ for $x_{0} \rightarrow \infty$ while functions in the set $D\left(\bar{H}_{\mathrm{rt}}\right)$ decay faster. 


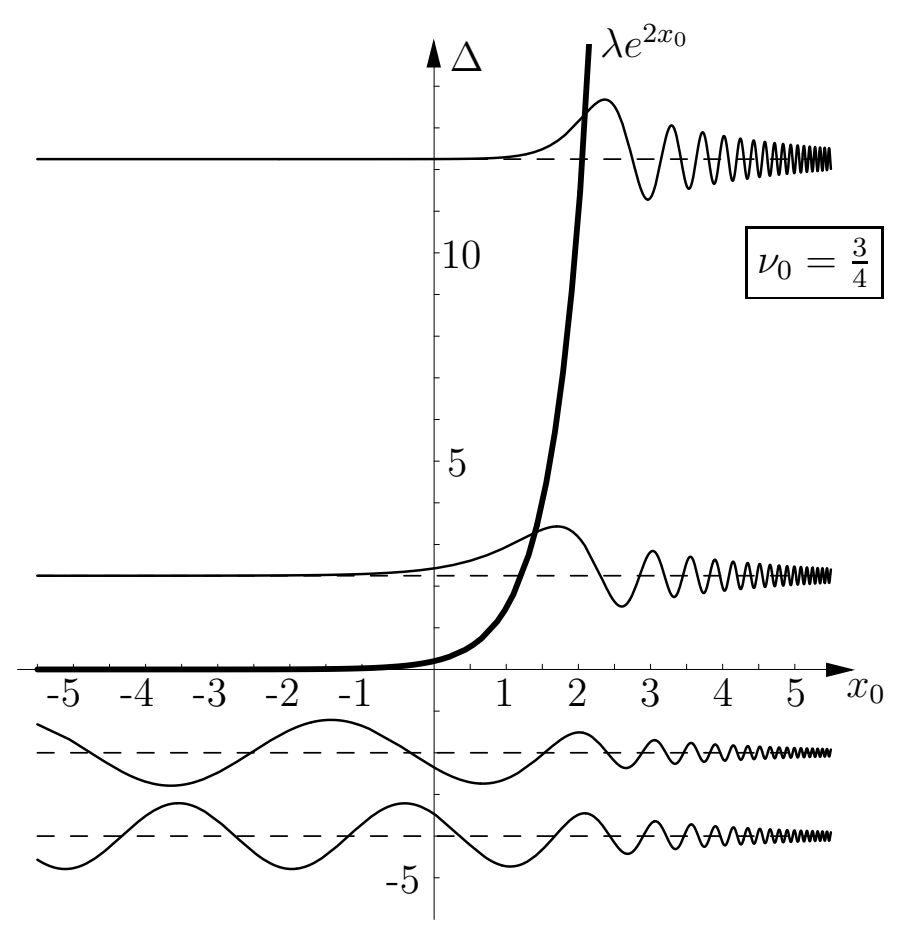

Figure 1: An illustration of the spectrum of the rolling tachyon model with $\lambda=0.2$ and boundary parameter $\nu_{0}=3 / 4$. For $\Delta>0$ the spectrum is discrete and we plotted the wave functions $\psi_{\nu_{n}}^{\nu_{0}}$ for $n=0,1$. The spectrum is continous for $\Delta<0$ and two wave functions $\psi_{\omega}^{\nu_{0}}$ are shown as representatives.

The asymptotic behaviour of functions in $D_{\nu^{\prime}}\left(H_{\mathrm{rt}}\right)$ is thus governed by the chosen linear combination $\psi_{1}^{-}+e^{2 \pi i \nu^{\prime}} \psi_{2}^{+}$. To determine the spectrum of $H_{\mathrm{rt}}$, we only have to analyse which solutions of eq. (2.4) have this specific asymptotic behaviour parametrised by $\nu^{\prime}$. The result agrees with the analysis we presented before and there is a one-to-one mapping from $\nu_{0}$ to $\nu^{\prime}$.

\subsection{Correlation functions for the rolling tachyon}

In this subsection we want to derive expressions for the minisuperspace analogue of 2- and 3 -point functions in the conformal field theory. We shall begin with the 3-point functions and then recover the simpler 2-point function as a special case. To make contact with standard conventions in Liouville field theory we choose a different normalization for our eigenfunctions with $\Delta \leq 0$,

$$
\tilde{\psi}_{\omega}^{\nu_{0}}\left(x_{0}\right)=(\lambda / 4)^{i \omega} \Gamma(1-2 i \omega)\left(J_{-2 i \omega}\left(\sqrt{\lambda} e^{x_{0}}\right)+\frac{\sinh \pi\left(\omega-i \nu_{0}\right)}{\sinh \pi\left(\omega+i \nu_{0}\right)} J_{2 i \omega}\left(\sqrt{\lambda} e^{x_{0}}\right)\right) .
$$


These wave functions possess the following asymptotic behaviour for $x_{0} \rightarrow-\infty$

$$
\tilde{\psi}_{\omega}^{\nu_{0}} \sim e^{-2 i \omega x_{0}}+R_{0}^{\nu_{0}}(\omega) e^{2 i \omega x_{0}} \quad ; R_{0}^{\nu_{0}}(\omega)=\left(\frac{\lambda}{4}\right)^{2 i \omega} \frac{\Gamma(1-2 i \omega)}{\Gamma(1+2 i \omega)} \frac{\sinh \pi\left(\omega-i \nu_{0}\right)}{\sinh \pi\left(\omega+i \nu_{0}\right)} .
$$

For obvious reasons, the quantity $R_{0}^{\nu_{0}}(\omega)$ is usually referred to as the reflection amplitude.

We can now calculate the three-point function in the minisuperspace approximation,

$$
C_{0}^{\nu_{0}}\left(\omega_{1}, \omega_{2}, \omega_{3}\right)=\int_{-\infty}^{\infty} d x_{0} \tilde{\psi}_{\omega_{1}}^{\nu_{0}}\left(x_{0}\right) e^{-2 i \omega_{2} x_{0}} \tilde{\psi}_{\omega_{3}}^{\nu_{0}}\left(x_{0}\right)=(\lambda / 4)^{2 i \tilde{\omega}} P_{0}^{\nu_{0}}\left(\omega_{j}\right) e^{Q_{0}\left(\omega_{j}\right)}
$$

where

$$
\exp Q_{0}\left(\omega_{1}, \omega_{2}, \omega_{3}\right)=\frac{1}{\Gamma(1+2 i \tilde{\omega})} \prod_{j=1}^{3} \frac{\Gamma\left(1+(-1)^{j} 2 i \omega_{j}\right)}{\Gamma\left(1-(-1)^{j} 2 i \tilde{\omega}_{j}\right)}
$$

and

$$
P_{0}^{\nu_{0}}\left(\omega_{1}, \omega_{2}, \omega_{3}\right)=\frac{i \pi}{2}\left[\frac{1}{\sinh 2 \pi \tilde{\omega}}+\frac{\zeta^{\nu_{0}}\left(\omega_{1}\right)}{\sinh 2 \pi \tilde{\omega}_{1}}-\frac{\zeta^{\nu_{0}}\left(\omega_{1}\right) \zeta^{\nu_{0}}\left(\omega_{3}\right)}{\sinh 2 \pi \tilde{\omega}_{2}}+\frac{\zeta^{\nu_{0}}\left(\omega_{3}\right)}{\sinh 2 \pi \tilde{\omega}_{3}}\right] .
$$

Here, we denoted by $\zeta^{\nu_{0}}(\omega)$ the relative phase in our combination of the two Bessel functions,

$$
\zeta^{\nu_{0}}(\omega)=\frac{\sinh \pi\left(\omega-i \nu_{0}\right)}{\sinh \pi\left(\omega+i \nu_{0}\right)}
$$

and we introduced the quantities $\tilde{\omega}, \tilde{\omega}_{j}$ through

$$
2 \tilde{\omega}=\omega_{1}+\omega_{2}+\omega_{3} \quad \tilde{\omega}_{j}=\tilde{\omega}-\omega_{j} .
$$

As explained in [12] we can recover the 2-point function of the minisuperspace model from $C_{0}^{\nu_{0}}$ by sending $\omega_{2}$ to zero,

$$
\lim _{\omega_{2} \rightarrow 0} C_{0}^{\nu_{0}}\left(\omega_{1}, \omega_{2}, \omega_{3}\right)=\pi R_{0}^{\nu_{0}}\left(\omega_{1}\right) \delta\left(\omega_{1}-\omega_{3}\right)
$$

where $R_{0}^{\nu_{0}}(\omega)$ was defined in eq. (2.8) above and we assume $\omega_{1}, \omega_{3} \geq 0$ as before. A comparison with the corresponding formulas in [12] shows that the features of our minisuperspace analysis are quite distinct from the minisuperspace model proposed by Strominger. In fact, we recover quantities of the latter by replacing our phases $\zeta^{\nu_{0}}(\omega)$ with the real function $[-\exp (-2 \pi \omega)]$. It might be interesting to observe that this replacement can be thought of as resulting from an integration over the parameter $\nu_{0}$. More precisely, using the integral formula

$$
\int_{0}^{1} d \nu_{0} \prod_{j=1}^{n} \zeta^{\nu_{0}}\left(\omega_{j}\right)=\prod_{j=1}^{n}\left(-e^{-2 \pi \omega_{j}}\right) \quad \text { for } \omega_{j}>0
$$


one can show that $C_{0}=\int d \nu_{0} C_{0}^{\nu_{0}}$ and $R_{0}=\int d \nu_{0} R_{0}^{\nu_{0}}$ agree with the 3 - and 2-point functions of Strominger's minisuperspace model for the rolling tachyon (see [12]). The latter was obtained from the minisuperspace theory of the usual Liouville model by a Wick rotation.

\section{The bouncing tachyon model}

We now proceed to the minisuperspace analysis of the bouncing tachyon model. Our discussion starts with a few remarks on the classical theory before we enter the spectral analysis of the bouncing tachyon Hamiltonian. Though our formulas will not be as explicit as in the case of the rolling tachyon model, we shall be able to argue that there is a 2parameter family of self-adjoint extensions which are adequate for the physics we want to study. All these extensions possess a discrete spectrum, both for positive and for negative eigenvalues.

\subsection{On the classical physics of bouncing tachyons}

The minisuperspace toy model for the bouncing tachyon background is obtained from the corresponding 2-dimensional field theory as in the case of the rolling tachyon. In terms of closed string parameters, the classical action reads

$$
S_{\mathrm{bt}}^{c}=-\int_{-\infty}^{\infty} d t\left(\frac{1}{4} \partial_{t} x_{0} \partial_{t} x_{0}+2 \lambda \cosh 2 x_{0}\right) .
$$

It is identical to the minisuperspace toy model for strings in an S-brane background up to a trivial change of parameters (see our discussion in section 2.1).

The key features of the classical theory can be deduced immediately from our discussion of the rolling tachyon model. Most importantly, it is easy to see that any solution of the bouncing tachyon theory reaches either $x_{0}=-\infty$ or $x_{0}=\infty$ in finite 'world-sheet' time $t_{f}$. This property of the bouncing tachyon theory suggests that the quantum mechanical model requires to fix boundary conditions in the far past and the far future. We shall prove this in the next subsection. Moreover, because the model now appears to be cut off at both sides, we expect the spectrum to be discrete even for $\Delta \leq 2 \lambda$. Since this is a new feature of the bouncing tachyon model, we would like to explore it more qualitatively and derive an explicit expression for the semi-classical spectral density. 
The basic observation is that the space of classical orbits with energies between $\Delta_{0}$ and $\Delta$ has a finite volume $\Gamma\left(\Delta_{0}, \Delta\right)$. We expect the number $N\left(\Delta_{0}, \Delta\right)$ of quantum mechanical states in the energy interval $\left(\Delta_{0}, \Delta\right)$ to be the corresponding classical volume in phase-space divided by $h=2 \pi \hbar=2 \pi{ }^{3}$

$$
N\left(\Delta_{0}, \Delta\right) \approx \frac{1}{2 \pi} \Gamma\left(\Delta_{0}, \Delta\right) .
$$

In the following we keep $\Delta_{0}$ constant as a reference energy and investigate the dependence of the volume $\Gamma$ on $\Delta$. For energies $\Delta_{0}<\Delta<2 \lambda$ the volume in phase-space is given by

$$
\Gamma_{-}\left(\Delta_{0}, \Delta\right)=2 \int_{-\infty}^{\infty}\left(\sqrt{2 \lambda \cosh 2 x_{0}-\Delta_{0}}-\sqrt{2 \lambda \cosh 2 x_{0}-\Delta}\right) d x_{0} .
$$

The integral is not difficult to evaluate and we obtain

$$
\Gamma_{-}\left(\Delta_{0}, \Delta\right)=c\left(\Delta_{0}\right)+4 \sqrt{2 \lambda-\Delta}\left(E\left(\sqrt{\frac{\Delta+2 \lambda}{\Delta-2 \lambda}}\right)-K\left(\sqrt{\frac{\Delta+2 \lambda}{\Delta-2 \lambda}}\right)\right) .
$$

Here, $c$ is independent of $\Delta$ and therefore it is irrelevant for our purposes. $K$ and $E$ are the complete elliptic integrals of first and second order, respectively. For any pair $\Delta_{0}, \Delta>-\infty$ this volume $\Gamma$ is finite. A similar calculation can be performed for $\Delta>2 \lambda$. In this case we find

$$
\Gamma_{+}\left(\Delta_{0}, \Delta\right)=c\left(\Delta_{0}\right)+4 \sqrt{2 \lambda+\Delta} E\left(\sqrt{1-\frac{\Delta-2 \lambda}{\Delta+2 \lambda}}\right) .
$$

The dependence of the phase-space volume on the energy $\Delta$ is shown in figure 2 ,

Semi-classical quantities are expected to provide good approximations for the exact quantum theory when $|\Delta|$ becomes large. Hence, we shall use our formulas to find the density of states for $|\Delta| \gg \lambda$. In the limit $\Delta \rightarrow-\infty$ we can estimate the dependence (3.11) of the volume $\Gamma_{-}$on $\Delta$ by

$$
\Gamma_{-}\left(\Delta_{0}, \Delta\right)=c\left(\Delta_{0}\right)-2 \sqrt{-\Delta}(\log (-4 \Delta / \lambda)-2)+\mathcal{O}\left(\frac{\log (-\Delta)}{\sqrt{-\Delta}}\right) .
$$

From the semi-classical rule (3.10) we may read off the size $\delta \Delta$ of the interval in which we find one quantum mechanical state, i.e. the level spacing

$$
\delta \Delta \approx 2 \pi\left(\frac{d}{d \Delta} \Gamma_{-}\left(\Delta_{0}, \Delta\right)\right)^{-1} \approx \frac{2 \pi \sqrt{-\Delta}}{\log (-4 \Delta / \lambda)} \text { for } \Delta \ll-\lambda .
$$

\footnotetext{
${ }^{3}$ Throughout this paper we use units in which $\hbar=1$.
} 
Our quantum mechanical models will later be shown to reproduce this result. A similar analysis carries through in the limit where $\Delta \rightarrow+\infty$. In fact, for large $\Delta$ we can approximate the value (3.12) of the phase-space volume $\Gamma_{+}$by

$$
\Gamma_{+}\left(\Delta_{0}, \Delta\right)=c\left(\Delta_{0}\right)+2 \pi \sqrt{\Delta}+\mathcal{O}\left(\frac{1}{\sqrt{\Delta}}\right)
$$

The level spacing is therefore

$$
\delta \Delta \approx 2 \sqrt{\Delta} \text { for } \Delta \gg \lambda
$$

This concludes our analysis of the semi-classical limit for the bouncing tachyon model.

\subsection{Spectral analysis of the bouncing tachyon}

In the following subsection we proceed to the quantum theory of the bouncing tachyon toy model, i.e. we investigate the 1-dimensional Hamilton operator

$$
H_{\mathrm{bt}}=\partial_{x_{0}}^{2}+2 \lambda \cosh 2 x_{0}
$$

As we have argued on the basis of the classical theory, we have to impose certain asymptotic boundary conditions on the wave functions. We shall see that when defined on the smooth functions with compact support, the Hamilton operator is not essentially selfadjoint. Instead, there is a 4-parameter family of self-adjoint extensions. In all of these extensions we have a purely discrete spectrum. Out of the 4-parameter family of extensions we are only interested in those where the boundary condition in the far future does not feed particles back into the far past. The precise formulation of this intuitive idea is shown below to single out a 2-parameter family of extensions, one parameter being associated to each boundary.

In the quantum mechanical problem the main task is to find appropriate domains of the Hamilton operator $H_{\mathrm{bt}}$. Let us first show that on smooth functions with compact support the operator is not essentially self-adjoint. We determine its deficiency indices by looking for square-integrable solutions $\psi$ of the differential equations $H_{\mathrm{bt}} \psi= \pm i \psi$. For each sign there are two linearly independent solutions. The asymptotics of these solutions for $x_{0} \rightarrow \pm \infty$ are the same as for the rolling tachyon in the limit $x_{0} \rightarrow \infty$, i.e. they fall off with an exponential suppression $\sim e^{-\left|x_{0}\right| / 2}$. Obviously, these functions are square-integrable and our deficiency indices are $(2,2)$. Standard operator theory then tells 
us that there is a family of self-adjoint extensions $D_{U}\left(H_{\mathrm{bt}}\right)$ labeled by the set of unitary $2 \times 2$-matrices $U$. Such matrices are parametrised by four independent parameters.

For physical reasons we are not interested in all possible extensions. Since we interpret $x_{0}= \pm \infty$ as far past and far future, we want independent conditions in the two limits. This means to replace a boundary condition of the form (2.3) by two separate conditions, one for each boundary

$$
\lim _{x_{0} \rightarrow \pm \infty}\left(\bar{\psi}\left(x_{0}\right) \partial_{x_{0}} \psi^{\prime}\left(x_{0}\right)-\psi^{\prime}\left(x_{0}\right) \partial_{x_{0}} \bar{\psi}\left(x_{0}\right)\right)=0 \quad \text { for all } \quad \psi, \psi^{\prime} \in D_{U}\left(H_{\mathrm{bt}}\right)
$$

Obviously, these new requirements on the asymptotics of wave functions are stronger than the symmetry condition (2.3). In fact, they reduce the number of parameters from four to two.

In finding the possible extensions, we can profit from our experience in the rolling tachyon scenario. There, we had to include functions $\psi$ into the domain of the Hamiltonian which behave asymptotically as

$$
\psi\left(x_{0}\right) \sim \text { const. } \cdot e^{-x_{0} / 2} \cos \left(\sqrt{\lambda} e^{x_{0}}-\frac{\pi}{4}-s\right)
$$

with some shift $s$. In the rolling tachyon model this parameter had to be the same real number $s=\pi \nu_{0}$ for all functions in the domain. Here we obtain a similar result: if we want to include functions with an asymptotic behaviour given in (3.17) for $x_{0} \rightarrow \infty$, it follows from eq. (3.16) that the shift $s=\pi \nu_{+}$has to be real and the same for all functions. This applies analogously for the asymptotics at $x_{0} \rightarrow-\infty$ with a possibly different boundary parameter $\nu_{-}$. We thus find domains $D_{\nu_{+}, \nu_{-}}\left(H_{\mathrm{bt}}\right)$ on which $H_{\mathrm{bt}}$ is symmetric.

It is now fairly easy to see that $H_{\mathrm{bt}}$ on such a domain is essentially self-adjoint. What we have to decide is whether there are functions solving the differential equations $H_{\mathrm{bt}} \psi^{ \pm}= \pm i \psi^{ \pm}$which are in the domain of the adjoint operator, i.e. whether there exist solutions $\psi^{ \pm}$satisfying

$$
\left(H_{\mathrm{bt}} \psi^{ \pm}, \phi\right)=\left(\psi^{ \pm}, H_{\mathrm{bt}} \phi\right) \quad \text { for all } \quad \phi \in D_{\nu_{+}, \nu_{-}}\left(H_{\mathrm{bt}}\right)
$$

From this condition we find that such $\psi^{ \pm}$have to have the same asymptotic behaviour as the functions in the domain $D_{\nu_{+}, \nu_{-}}\left(H_{\mathrm{bt}}\right)$. It is not hard to see that these asymptotics are incompatible with the differential equations for $\psi^{ \pm}$. Hence, the deficiency indices of the bouncing tachyon Hamiltonian on our extended domains are zero. 
Having found the right domains, we can start to analyse the spectrum. The differential equation we have to solve when we look for eigenfunctions $\psi_{\Delta}\left(x_{0}\right)$ of (3.15) is known under the name 'modified Mathieu equation' (see e.g. [21]),

$$
\psi_{\Delta}^{\prime \prime}\left(x_{0}\right)+2 \lambda \cosh \left(2 x_{0}\right) \psi_{\Delta}\left(x_{0}\right)=\Delta \psi_{\Delta}\left(x_{0}\right)
$$

Its solutions are given by the modified Mathieu functions of the first kind $\mathrm{Ce}_{\nu}\left(x_{0}, \lambda\right)$ and $\operatorname{Se}_{\nu}\left(x_{0}, \lambda\right)$ which are even and odd under $x_{0} \rightarrow-x_{0}$, respectively. The parameter $\nu=\nu(\Delta, \lambda)$ is called a characteristic exponent to $\Delta$ and $\lambda$. Note that the statements we have just made are only true if $\nu$ is not an integer. Since the whole story gets a lot more involved for integer characteristic exponent $\nu$ (see e.g. [21]), we assume throughout our discussion that $\nu$ is non-integer. For large values of $|\Delta|$ the characteristic exponent $\nu$ is related with the eigenvalue $\Delta$ by $\Delta \approx \nu^{2}$. To analyse the asymptotic behaviour of the eigenfunctions, the following linear combinations are convenient

$$
\mathrm{Me}_{ \pm \nu}\left(x_{0}, \lambda\right)=\mathrm{Ce}_{\nu}\left(x_{0}, \lambda\right) \pm \operatorname{Se}_{\nu}\left(x_{0}, \lambda\right)
$$

When $\left|x_{0}\right|$ is large, they behave like Bessel functions,

$$
\begin{aligned}
\operatorname{Me}_{\nu}\left(x_{0}, \lambda\right) & \sim \frac{\operatorname{Me}_{\nu}(0, \lambda)}{\mathrm{M}_{\nu}^{(1)}(0, \lambda)} J_{\nu}\left(\sqrt{\lambda} e^{x_{0}}\right) \quad \text { for } \quad x_{0} \rightarrow \infty \\
\operatorname{Me}_{\nu}\left(x_{0}, \lambda\right) & \sim \frac{\operatorname{Me}_{\nu}(0, \lambda)}{\mathrm{M}_{-\nu}^{(1)}(0, \lambda)} J_{-\nu}\left(\sqrt{\lambda} e^{\left|x_{0}\right|}\right) \quad \text { for } \quad x_{0} \rightarrow-\infty .
\end{aligned}
$$

Expressions for $\mathrm{M}_{ \pm \nu}^{(1)}(0, \lambda)$ can be found in [21, Chapter 2]. Here we shall only need the approximation for the ratio

$$
\xi_{\nu}(\lambda):=\frac{\mathrm{M}_{\nu}^{(1)}(0, \lambda)}{\mathrm{M}_{-\nu}^{(1)}(0, \lambda)}=\left(\frac{\lambda}{4}\right)^{\nu} \frac{\Gamma(1-\nu)}{\Gamma(1+\nu)}\left(1+\mathcal{O}\left(\lambda^{2} / \nu\right)\right) .
$$

Note that this approximation is not valid when $\nu$ approaches an integer number. ${ }^{4}$ Having gathered some background information we are now prepared to study eigenfunctions of our Hamiltonian. For such eigenfunctions we make an Ansatz of the form

$$
\psi_{\nu}\left(x_{0}, \lambda\right)=a \mathrm{Me}_{\nu}\left(x_{0}, \lambda\right)+b \mathrm{Me}_{-\nu}\left(x_{0}, \lambda\right) .
$$

\footnotetext{
${ }^{4}$ If $\nu$ is real, the error estimate in eq. (3.18) is still correct if we keep the (non-zero) fractional part of $\nu$ fixed while letting $|\nu|$ grow.
} 
Using such an Ansatz, our boundary conditions with labels $\nu_{+}, \nu_{-}$turn into two constraints for the three parameters $a, b$ and $\nu$,

$$
\begin{aligned}
& a \sin \pi\left(\nu_{+}-\frac{\nu}{2}\right)+b \xi_{\nu}(\lambda) \sin \pi\left(\nu_{+}+\frac{\nu}{2}\right)=0 \\
& a \xi_{\nu}(\lambda) \sin \pi\left(\nu_{-}+\frac{\nu}{2}\right)+b \sin \pi\left(\nu_{-}-\frac{\nu}{2}\right)=0
\end{aligned}
$$

A non-trivial solution of these constraints exists whenever the following equation is fulfilled,

$$
\sin \pi\left(\nu_{+}-\frac{\nu}{2}\right) \sin \pi\left(\nu_{-}-\frac{\nu}{2}\right)=\xi_{\nu}^{2}(\lambda) \sin \pi\left(\nu_{+}+\frac{\nu}{2}\right) \sin \pi\left(\nu_{-}+\frac{\nu}{2}\right) .
$$

This condition determines the spectrum of $H_{\mathrm{bt}}$. It can only be satisfied for isolated values of $\nu$ and therefore we find a discrete spectrum. The spectrum and the corresponding wave functions are sketched in figure 3, For generic values of $\nu_{+}, \nu_{-}$, eq. (3.19) cannot be solved explicitly. Our aim now is to determine the spectrum approximately in the limit $|\Delta| \rightarrow \infty$.

Let us first consider the case $\Delta \rightarrow+\infty$, i.e. $\nu \rightarrow \infty$. Based on our semi-classical analysis in section 3.1 we expect an average level spacing of $\delta \nu=1$ (cf. eq. (3.14)). In the exact quantum theory we conclude from eq. (3.18) that $\xi_{\nu}(\lambda)$ vanishes in the limit $\nu \rightarrow \infty$, at least when $\nu$ is not close to integers. Hence, the condition (3.19) turns into the simple relation

$$
\sin \pi\left(\nu_{+}-\frac{\nu}{2}\right) \sin \pi\left(\nu_{-}-\frac{\nu}{2}\right)=0
$$

which has the solutions

$$
\nu=2\left(\nu_{+}+n\right) \quad \text { or } \quad \nu=2\left(\nu_{-}+n\right) \quad \text { with } n \in \mathbb{N}
$$

Let us remark that in the special case $\nu_{+}=1-\nu_{-}$, this solution becomes the exact spectrum for real $\nu$. The spectrum in eq. (3.20) is just the union of the spectra of two Liouville theories with boundary parameters $\nu_{+}$and $\nu_{-}$. Indeed we obtain an average level spacing of $\delta \nu=1$. In our analysis we found it hard to control the case when $\nu$ comes very close to an integer number, but the comparison with the semi-classical expectations tells us that we already found all eigenvalues.

Now we want to analyse the limit $\Delta \rightarrow-\infty$. If we set $\nu=i \omega$, we have to study the limit $\omega \rightarrow \infty$. From the condition (3.19) we obtain

$$
\frac{1+\xi_{i \omega}^{2}(\lambda)}{1-\xi_{i \omega}^{2}(\lambda)}=i \frac{1-\tan \pi \nu_{+} \tan \pi \nu_{-}}{\tan \pi \nu_{+}+\tan \pi \nu_{-}}+\mathcal{O}\left(e^{-\pi \omega}\right) \text {. }
$$




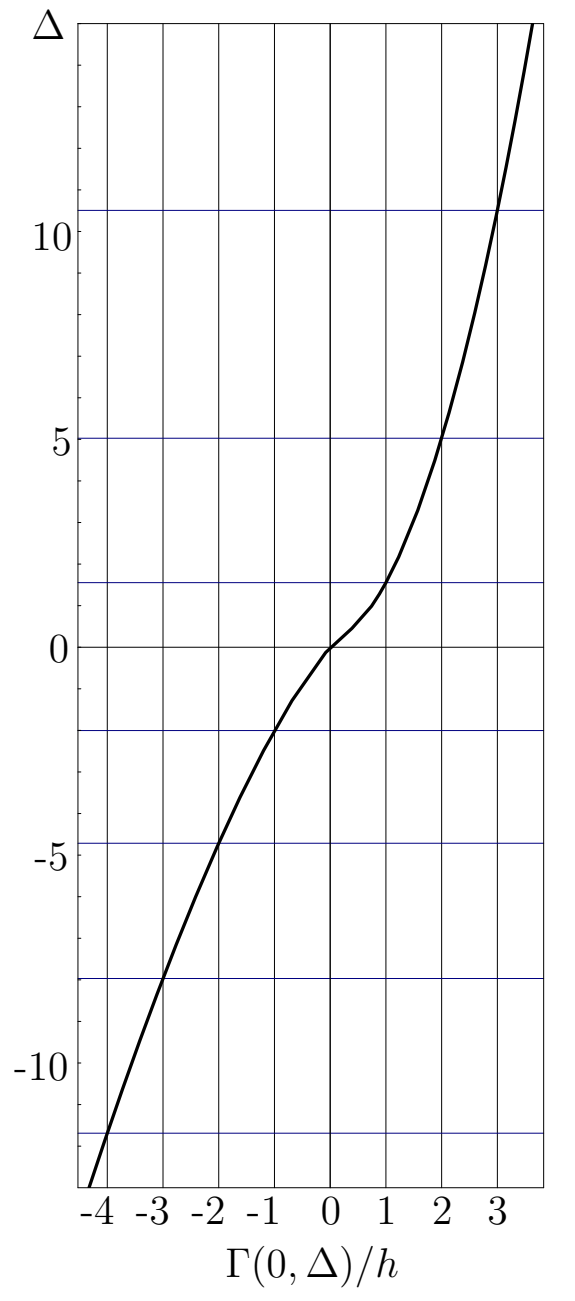

Figure 2: Energy $\Delta$ versus phasespace volume $\Gamma(0, \Delta)$. Neighbouring grid lines correspond to a spacing $\delta \Gamma=h$, i.e. in the energy interval $\delta \Delta$ between two horizontal grid lines we expect to find one quantum mechanical state.

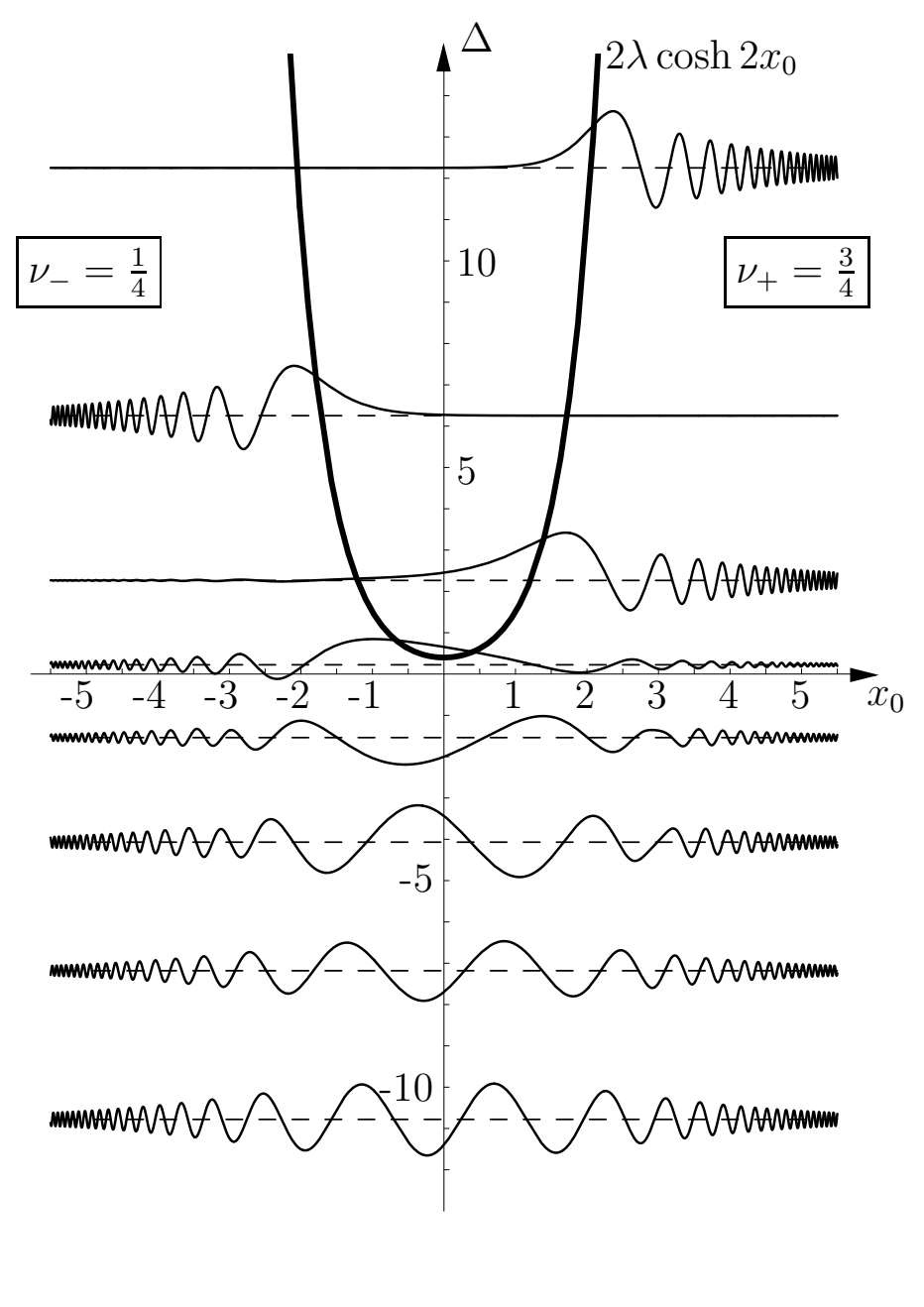

Figure 3: An illustration of the spectrum of the bouncing tachyon model. The boundary labels are $\nu_{+}=3 / 4$ and $\nu_{-}=1 / 4$, and we set $\lambda=0.2$. The spectrum is purely discrete, and the level spacing agrees well with the semi-classical expectations as we can see by comparing with the neighbouring figure 2. The right half of the drawing resembles strongly the figure of the rolling tachyon model (fig. 11). 
For large values of $\omega$ we can approximate $\xi_{i \omega}(\lambda)$ using eq. (3.18) and Stirling's formula,

$$
\xi_{i \omega}(\lambda)=-i e^{i \omega\left(\log \frac{\lambda}{4 \omega^{2}}+2\right)}(1+\mathcal{O}(1 / \omega)) .
$$

If we substitute this expression into eq. (3.21), we find that the spectrum of large negative $\Delta=-\omega^{2}$ is determined by the equation

$$
\omega\left(\log \left(4 \omega^{2} / \lambda\right)-2\right)+\mathcal{O}(1 / \omega)=\pi n+\arctan \frac{\tan \pi \nu_{+} \tan \pi \nu_{-}-1}{\tan \pi \nu_{+}+\tan \pi \nu_{-}}
$$

with $n \in \mathbb{N}$. The spacing $\delta \omega$ between two solutions of this equation takes the form

$$
\delta \omega \approx \pi\left(\log \left(4 \omega^{2} / \lambda\right)\right)^{-1} .
$$

When we translate this into the energy difference $\delta \Delta=2 \sqrt{-\Delta} \delta \omega$ between two eigenvalues, we recover the semi-classical result (3.13).

\section{Conclusions and open problems}

Above we have analysed the minisuperspace model of rolling and bouncing tachyons, i.e. of time-like Liouville and sine-Gordon theory. In contrast to the more complicated field theories, their minisuperspace toy models are easily treated directly, without performing a Wick rotation from the corresponding Euclidean background. The results we obtained are certainly very suggestive of several features in the field theory models. In particular, we expect that time-like (boundary) Liouville theory comes with one real parameter $\nu_{0}$ which describes some boundary condition in the far future $x_{0}=\infty$. The latter must be imposed because in an exponentially unbounded potential, particles and strings reach infinity in finite world-sheet time. Technically, the parameter comes in through the need to make the generator of world-sheet time translations self-adjoint. We have made similar statements about the time-like (boundary) sine-Gordon model only that in this case a 2-parameter family is predicted from the minisuperspace analysis. Each member of this family can be argued to possess a purely discrete spectrum of conformal weights. Finally, we saw that our two toy models contain a discrete set of states with positive eigenvalue $\Delta$. It remains to be seen whether the associated field theories also possess primaries of arbitrarily large conformal weight, or whether their spectrum gets truncated.

The construction of the full field theory models is certainly a very interesting open problem. Progress in this direction could possibly be made along different lines. Inspired 
by our observation that minisuperspace quantities in our rolling tachyon models are obtained from the Wick-rotated theory by a replacement $\exp (-2 \pi \omega) \rightarrow-\zeta^{\nu_{0}}(\omega)$, one might try to start from the known expression for bulk 3-point functions in the Wick-rotated $c=1$ Liouville theory [12] (see also [22] for earlier expressions of the bulk 2-point functions) and proceed to their $\nu_{0}$-dependent 'components' by some kind of 'unitarisation'. Another, maybe more promising approach could pass through path integral representations of the time-like model. Using arguments as in [23, this might lead to modified screening integrals and then ultimately to new $\nu_{0}$-dependent solutions of time-like Liouville theory, very much along the lines of [24, 25, 26]. We plan to come back to these problems in the near future.

While our results above allow to draw rather obvious and well motivated conclusions for the involved conformal field theories, it seems less clear that the parameters $\nu_{0}$ or $\nu_{ \pm}$ will also show up in string theory amplitudes. In string theory, the Hamiltonian of the underlying world-sheet theory is only used to formulate the physical state condition, i.e. it appears in the form of a constraint. Even though the usual techniques to solve such constraints do also exploit self-adjointness, this may not stand up as a firm argument against other scenarios in which string theory amplitudes involve some averaging over different CFT backgrounds. Actually, there are several examples of dynamical processes in string theory which are believed to end in a 'mixed' final state. This is particularly well established for the condensation of an open string tachyon which can cause a single brane to decay into a final configuration containing several lower dimensional branes. Examples of mixed final states that arise from the condensation of closed string tachyons can be found e.g. in [27]. Clues on the role of the parameters $\nu_{0}$ and $\nu_{ \pm}$in string theory might come from more thorough investigations of quantum field theories with time dependent masses or from the duality between closed strings in a flat background and open strings on decaying branes (see e.g. [28] for some recent study of this duality). We believe that this issue deserves further investigation.

Acknowledgements: We would like to thank R. Kashaev for a discussion that initiated this work and for sharing results of a collaboration with L.D. Faddeev and M. Volkov. Moreover, we are indebted to A.Yu. Alekseev, M. Berkooz, K. Fredenhagen, H. Grosse, S. Ribault, A. Sen, J. Teschner and T. Thiemann for very useful conversations. 


\section{A Completeness}

Our aim in this appendix is to show that the set of eigenfunctions $\psi_{\nu_{n}}^{\nu_{0}}, \psi_{\omega}^{\nu_{0}}$ (see eqs. $(2.52 .6)$ ) is complete, i.e. that eq. (2.7) is fulfilled.

We start with the integral

$$
I\left(\alpha, \alpha^{\prime}\right)=\int_{0}^{\infty} d \omega \overline{\psi_{\omega}^{\nu_{0}}}(\alpha) \psi_{\omega}^{\nu_{0}}\left(\alpha^{\prime}\right)
$$

where $\alpha=\sqrt{\lambda} e^{x_{0}}, \alpha^{\prime}=\sqrt{\lambda} e^{x_{0}^{\prime}}$. We insert the expression (2.6) for $\psi_{\omega}^{\nu_{0}}$ and obtain

$$
\begin{aligned}
I\left(\alpha, \alpha^{\prime}\right)=2 \int_{0}^{\infty} d \omega \frac{\omega}{\sinh 2 \pi \omega} & \left(J_{2 i \omega}(\alpha) J_{-2 i \omega}\left(\alpha^{\prime}\right)+J_{-2 i \omega}(\alpha) J_{2 i \omega}\left(\alpha^{\prime}\right)\right. \\
& \left.+\zeta^{\nu_{0}}(-\omega) J_{-2 i \omega}(\alpha) J_{-2 i \omega}\left(\alpha^{\prime}\right)+\zeta^{\nu_{0}}(\omega) J_{2 i \omega}(\alpha) J_{-2 i \omega}\left(\alpha^{\prime}\right)\right) .
\end{aligned}
$$

Here, $\zeta^{\nu_{0}}(\omega)$ is defined in eq. (2.9) . We expect this integral to be a distribution, so we introduce an extra factor $1 /\left(\varepsilon^{2} \omega^{2}+1\right)$ in the integral to regularize it. We allow $\varepsilon$ to be complex with positive real part. At the end we shall take the limit $\varepsilon \rightarrow 0$.

Let us assume that $\alpha \geq \alpha^{\prime}$. We evaluate the integral by rewriting it first as an integral over the whole real line,

$I_{\varepsilon}\left(\alpha, \alpha^{\prime}\right)=2 \int_{-\infty}^{\infty} d \omega \frac{\omega}{\sinh 2 \pi \omega}\left(J_{2 i \omega}(\alpha) J_{-2 i \omega}\left(\alpha^{\prime}\right)+\zeta^{\nu_{0}}(-\omega) J_{-2 i \omega}(\alpha) J_{-2 i \omega}\left(\alpha^{\prime}\right)\right) \frac{1}{\varepsilon^{2} \omega^{2}+1}$.

By a careful analysis of the asymptotic behaviour of $J_{\nu}$ for large order $|\nu|$ we find that we can close the contour in the upper half-plane without any contribution from the semicircle at infinity. Using Cauchy's theorem, we can evaluate our integral by the sum of residues of poles in the upper half-plane. Note that there are no poles at the zeroes of $\sinh 2 \pi \omega$. The only poles come from $\zeta^{\nu_{0}}(-\omega)$ at $\omega=i\left(\nu_{0}+n\right), n \in \mathbb{N}^{*}$ and from our regulator at $\omega=i / \varepsilon$. We choose $\varepsilon$ s.t. the poles do not meet. The contribution from the residues at $\omega=i\left(\nu_{0}+n\right)$ is

$$
2 \pi i \sum(\text { Residues })=-4 \sum_{n=0}^{\infty}\left(\nu_{0}+n\right) J_{2\left(\nu_{0}+n\right)}(\alpha) J_{2\left(\nu_{0}+n\right)}\left(\alpha^{\prime}\right) \frac{1}{1-\varepsilon^{2}\left(n+\nu_{0}\right)^{2}} .
$$

In the limit $\varepsilon \rightarrow 0$ this approaches the contribution from the discrete spectrum.

It remains to evaluate the residue at $\omega=i / \varepsilon$ in the limit $\varepsilon \rightarrow 0$. This is a straightforward exercise in playing with the asymptotic behaviour of Bessel functions at large order (see e.g. [29]). We obtain at the end

$$
I\left(\alpha, \alpha^{\prime}\right)+\sum_{n=0}^{\infty} \overline{\psi_{\nu_{n}}^{\nu_{0}}}(\alpha) \psi_{\nu_{n}}^{\nu_{0}}\left(\alpha^{\prime}\right)=\lim _{\varepsilon \rightarrow 0} \frac{1}{\varepsilon} e^{-2\left|\log \left(\alpha / \alpha^{\prime}\right)\right| / \varepsilon}
$$


The right hand side is an expression for the delta-distribution $\delta\left(\log \left(\alpha / \alpha^{\prime}\right)\right)=\delta\left(x_{0}-x_{0}^{\prime}\right)$. This is what we wanted to show.

\section{References}

[1] M. Gutperle and A. Strominger, Spacelike branes, JHEP 0204 (2002) 018 arXiv:hep-th/0202210.

[2] A. Sen, Rolling tachyon, JHEP 04 (2002) 048 hep-th/0203211.

[3] A. Sen, Time evolution in open string theory, JHEP 10 (2002) 003 hep-th/0207105.

[4] A. Strominger, Open string creation by S-branes, hep-th/0209090.

[5] P. Mukhopadhyay and A. Sen, Decay of unstable D-branes with electric field, JHEP 11 (2002) 047 hep-th/0208142.

[6] T. Okuda and S. Sugimoto, Coupling of rolling tachyon to closed strings, Nucl. Phys. B647 (2002) 101-116 hep-th/0208196.

[7] B. Chen, M. Li and F.-L. Lin, Gravitational radiation of rolling tachyon, JHEP 11 (2002) 050 hep-th/0209222.

[8] S.-J. Rey and S. Sugimoto, Rolling tachyon with electric and magnetic fields: Tduality approach, Phys. Rev. D67 (2003) 086008 hep-th/0301049.

[9] F. Larsen, A. Naqvi and S. Terashima, Rolling tachyons and decaying branes, JHEP 02 (2003) 039 hep-th/0212248.

[10] N. Lambert, H. Liu and J. Maldacena, Closed strings from decaying D-branes, hep-th/0303139

[11] M. Gutperle, A. Strominger, Timelike boundary Liouville theory, Phys. Rev. D67 (2003) 126002 hep-th/0301038.

[12] V. Schomerus, Rolling tachyons from Liouville theory, arXiv:hep-th/0306026.

[13] T. Fulop, Reduced $S L(2, R)$ WZNW quantum mechanics, J. Math. Phys. 37 (1996) 1617 arXiv:hep-th/9502145. 
[14] H. Kobayashi and I. Tsutsui, Quantum mechanical Liouville model with attractive potential, Nucl. Phys. B 472 (1996) 409 arXiv:hep-th/9601111.

[15] J. Kluson, Particle production on half S-brane, arXiv:hep-th/0306002.

[16] J. Kluson, The Schrödinger wave functional and closed string rolling tachyon, arXiv:hep-th/0308023.

[17] A. Maloney, A. Strominger and X. Yin, S-brane thermodynamics, arXiv:hep-th/0302146.

[18] J. Kluson, The Schrödinger wave functional and S-branes, arXiv:hep-th/0307079.

[19] M. Reed and B. Simon, Methods of modern mathematical physics. Part II: Fourier analysis, self-adjointness, Academic Press, New York (1975)

[20] J. von Neumann, Allgemeine Eigenwerttheorie Hermitescher Funktionaloperatoren, Math. Ann. 102 (1929) 49

[21] J. Meixner and F.W. Schäfke, Mathieusche Funktionen und Sphäroidfunktionen, Grundlehren der Mathematische Wissenschaften 71, Springer, Berlin (1954)

[22] A. Strominger and T. Takayanagi, Correlators in timelike bulk Liouville theory, hep-th/0303221.

[23] M. Goulian and M. Li, Correlation functions in Liouville theory, Phys. Rev. Lett. 66 (1991) 2051-2055.

[24] J. Teschner, On the Liouville three point function, Phys. Lett. B363 (1995) 65-70 hep-th/9507109.

[25] V. Fateev, A. B. Zamolodchikov and A. B. Zamolodchikov, Boundary Liouville field theory. I: Boundary state and boundary two-point function, hep-th/0001012.

[26] A. B. Zamolodchikov and A. B. Zamolodchikov, Liouville field theory on a pseudosphere, hep-th/0101152.

[27] J. A. Harvey, D. Kutasov, E. J. Martinec and G. Moore, Localized tachyons and RG flows, arXiv:hep-th/0111154. 
[28] J. L. Karczmarek, H. Liu, J. Maldacena and A. Strominger, UV finite brane decay, arXiv:hep-th/0306132.

[29] G. Watson, A treatise on the theory of Bessel functions, Cambridge University Press, 2nd edition edition (1944) 